\title{
Factores asociados al rendimiento académico de estudiantes de Ingeniería Estadística, Universidad Nacional de Trujillo, 2019
}

\author{
Factors associated with the academic performance of Statistical \\ Engineering students, National University of Trujillo, 2019
}

\author{
${ }^{1}$ Noelia P. Rodriguez Paredes \\ ${ }^{2}$ Elmis J. García Zare \\ ${ }^{3}$ Luis A. López Puycan
}

ORCID: 0000-0002-3640-2779

ORCID: 0000-0003-4863-7230

ORCID: 0000-0003-4454-6737

\begin{abstract}
RESUMEN
El objetivo de la investigación se centró en determinar factores asociados al rendimiento académico, para ello, se usó un diseño no experimental, transversal y descriptivo. Se utilizóla técnica de muestreo aleatorio irrestricto para determinar el tamaño de muestra, dando como resultado 19 y 15 estudiantes de II Y IV ciclo respectivamente. Las técnicas estadísticas empleadas, utilizando el software R-Studio, fueron: la Selección de Clústeres para la clasificación de variables, donde se determinó 4 clúster denominados: Nivel educativo más alto entre los padres del estudiante, Factores Sociodemográficos, Medio de traslado a la universidad y Factores de distancia y responsabilidad académica; y el Análisis Factorial de Correspondencias Múltiples determinándose el perfil de los estudiantes con promedio ponderado de mayor frecuencia, comprendido de 11 a 14, el cual se caracteriza por: su conformación de grupos que es heterogénea, sexo femenino, no presentan cursos rezagados, su medio de traslado es el transporte público, viven en Trujillo Metropolitano y tienen una edad mayor o igual a 20 años.
\end{abstract}

Palabras Claves: Perfil de los estudiantes, rendimiento académico.

\begin{abstract}
The aim of the research was to determine the factors associated with academic performance. A non-experimental, cross-sectional and descriptive design was used. The unrestricted random sampling technique was used to determine the sample size, resulting in 19 and 15 students of the II and IV cycle respectively. The statistical techniques used, using the R-Studio software, were: Cluster Selection for the classification of variables, where 4 clusters were determined: Highest educational level among the student's parents, Sociodemographic factors, Modes of transportation to get to the university campus and Factors of distance and academic responsibility; and the Factorial Analysis of Multiple Correspondences, determining the profile of the students with the most frequent weighted average, comprised from 11 to 14 , which is characterized by: their composition of groups that is heterogeneous, female sex, they do not present lagging courses, they use the public transport, they live in Trujillo Metropolitano and are older than or equal to 20 years.
\end{abstract}

Keywords: Student profile, academic performance.

\footnotetext{
${ }^{1}$ Universidad Nacional de Trujillo. Trujillo, Perú. E-mail: nrodriguezp@ unitru.edu.pe

${ }^{2}$ Universidad Nacional de Trujillo. Trujillo, Perú. E-mail: egarciaz@ unitru.edu.pe

${ }^{3}$ Universidad Nacional Jorge Basadre Grohmann. Tacna, Perú. E-mail: llopezp@ unjbg.edu.pe
} 


\section{INTRODUCCIÓN}

Hoy en día nos encontramos en una situación académica muy exigente y aunque no todos los estudiantes llegan a realizar estudios superiores, los que lo logran se enfrentan a un panorama académico competitivo y a factores que incluso pueden llevarlos al fracaso. Los jóvenes pasan por diferentes etapas en su trayectoria universitaria y están sometidos a cambios que los lleva a adecuarse a un nuevo sistema que los forma para su ingreso a la vida laboral.

El problema más común de los estudiantes es que, a pesar de que tienen una oferta educativa amplia comparada con años anteriores, un mayor acceso a las nuevas tecnologías, y la disponibilidad de postular a una variedad de becas; esto no asegura una mejora académica, lo que trae como consecuencia problemas académicos (Chong, 2017). Asimismo, Abarca (2019) nos indica que "Haber reprobado cuatro veces una misma asignatura motivó el retiro definitivo de 118 estudiantes pertenecientes a la casa de estudios Universidad Nacional de San Agustín (UNSA)". Según la firma Penta Analytics (2017), en su estudio realizado encontró que "El 27 \% de ingresantes desertan en el primer año de la carrera de estudios. Los motivos que impulsan a que alumnos de pregrado abandonen su formación, se basan en cuatro factores principales: bajo rendimiento, problemas económicos, indecisión vocacional y problemas afectivos". Según datos del Minedu (2016), "El Perú se caracteriza por las mínimas posibilidades que tiene su población para permitirse una educación superior. De las personas entre 17 y 24 años, sólo el 42,7 \% de estos pueden dedicarse a una carrera universitaria o técnica profesional, lo cual representa tan solo a 1,9 millones" (p.138).

Teniendo en cuenta lo expuesto, si nos proyectamos a los años 2020 - 2021, se espera que los estudiantes de educación superior mejoren su nivel de rendimiento académico a comparación de los años anteriores gracias a la mejora del nuevo currículo de enseñanza superior basada en competencias donde el estudiante es el principal protagonista en la educación y su bienestar emocional es de vital importancia.

La realidad de los estudiantes de la Escuela de Estadística no es ajena a los inconvenientes de poco desarrollo académico, que es visto como problema mundial; existen una serie de factores que pueden estar afectando su formación académica, los que al no ser resueltos podrían afectar su proceso de formación continua e incluso trascender al ejercicio profesional. Por ello, se optó por realizar este estudio para identificar los elementos relacionados a su desempeño estudiantil. Esta investigación se fundamentó en los trabajos previos que se mencionan a continuación.

Martin, Sevilla y Jenaro (2018) desarrollaron una investigación para poder determinar los factores personales-institucionales incidentes en el desempeño académico en un curso de postgrado. Este estudio fue de enfoque cuantitativo. Los candidatos formaron parte de las cohortes 2004 al 2014, y fueron agrupados en estudiantes con niveles de bajo o alto rendimiento académico. Los datos para este estudio se recolectaron mediante un cuestionario estructurado que investigó sobre: desempeño del asesor y tutor, conocimientos anteriores, antecedentes académicos, diplomacia para laborar en grupos de trabajo, expectativas del curso, nivel de autoestima, estímulos, bienestar psicológico, procesos académicos y administrativos. Finalmente, se determinó que la trayectoria sobre indagación, estrategias de estudio y la propia estima personal se definen como aspectos 
esenciales que inciden en el éxito o fracaso académico de los estudiantes pertenecientes a dicho curso.

Además, Muradás (2016) realizó un estudio centrado en el diagnóstico de algunos factores de riesgo asociados al abandono de sus carreras. La población de estudio fueron los estudiantes de nuevo ingreso. El tipo de investigación llevado a cabo fue descriptivo. El método de investigación que se aplicó fue mixto: cualitativo y cuantitativo. Llegándose a sintetizar que las y los jóvenes al egresar del nivel medio no cuentan con los conocimientos previos necesarios en las asignaturas básicas, no estudiaron una especialidad de acuerdo a la ingeniería, por lo que, carecen de las bases en matemáticas e inglés, no tienen buenas relaciones interpersonales y existen pocos estudiantes que cuentan con herramientas tecnológicas suficientes que les apoyen en sus estudios, como internet y equipamiento.

Así también Solano (2015), midió la asociación entre el desempeño estudiantil de los pupilos con capacidades intelectuales y adecuación frente al estudio. El tipo de estudio fue correlacional con enfoque cuantitativo. Finalmente, encontró que asistir a clases con determinada motivación, la planificación y el método de trabajo están asociadas con el rendimiento $(r=0,560, r=0,423$ y $r=0,560$, respectivamente).

Por último, en la Universidad Nacional de Trujillo se realizó un estudio donde se comprobó que existen diferentes factores por los cuales los estudiantes de derecho del tercer ciclo presentaban un nivel bajo de rendimiento académico, entre ellas, se consideró que una de las principales razones por las que un porcentaje elevado de estudiantes tienen bajo rendimiento es por la falta de tiempo, debido a que ellos tienen que trabajar para generar ingresos y poder financiar sus estudios; otro de los factores considerados fue la mala organización y no cumplimiento de los horarios establecidos por la facultad; finalmente, un grupo de estudiantes considero que la enseñanza que se les imparte es regular y que esto influye en su rendimiento académico (Echevarría, 2013, p. 88 - 89).

Respecto a las bases teórico científicas tenemos a Garbanzo (2007) que, en el libro "Factores asociados al rendimiento académico en estudiantes universitarios", indicó que los mismos son multicausales, es decir, capturan una amplia capacidad explicativa de las distintas variables y el tiempo en función del espacio que toman parte del procedimiento enlazado entre la educación y el aprendizaje, clasificándolos en Factores personas, sociales e institucionales.

Para el presente estudio se formuló el siguiente problema de investigación. ¿Cuáles son los factores que se encuentran asociados al rendimiento académico de los estudiantes de Ingeniería Estadística de la Universidad Nacional de Trujillo, 2019? Esta investigación se justifica al ser útil para los docentes de educación superior universitaria enfocados realmente por el nivel de desempeño estudiantil de sus alumnos, capacitándose constantemente en nuevas técnicas y metodologías de enseñanza para llegar estratégicamente al alumno. La nueva modalidad de evaluación que es por competencias, conlleva al interés por el alumno por parte del docente no solo en la parte educativa, sino también en la parte actitudinal, pues esta investigación es un aporte para que los docentes conozcan los factores asociados con el rendimiento académico de los estudiantes, para que puedan reconocer la importancia de atender todos estos aspectos y lograr la mayor cantidad de estudiantes promovidos. 
El objetivo general de este trabajo es determinar los factores asociados al rendimiento académico de los estudiantes de Ingeniería Estadística de la Universidad Nacional de Trujillo, 2019. Los objetivos específicos son: clasificar los factores asociados al rendimiento académico de los estudiantes de Ingeniería Estadística de la Universidad Nacional de Trujillo, 2019 y describir el perfil de los estudiantes de Ingeniería Estadística de la Universidad Nacional de Trujillo, 2019.

La hipótesis que se plantea para responder al problema de investigación es implícita, dado que es una investigación descriptiva. Las técnicas multivariadas empleadas fueron el análisis de clúster y el de correspondencia múltiple. El primero, tiene como objetivo crear grupos lo más parecidos internamente y lo más diferentes entre ellos. Se parte de individuos caracterizados por variables, a partir de calcular medidas de similitud o de disimilitud (proximidad). Para la elección de la medida de proximidad; si son variables continuas se trabaja con la Distancia euclidiana:

$$
d\left(i, i^{\prime}\right)=d_{i i^{\prime}}=\sqrt{\sum_{j=1}^{p}\left(X_{i j}-X_{i^{\prime} j}\right)^{2}}
$$

Posteriormente, se elige el método de clasificación: jerárquico o no jerárquico, se realiza la clasificación en un número de grupos y se valida la tipología (Vidal, 2018).

El segundo método se basa en realizar una correspondencia sobre la llamada matriz de Burt: $Z^{\mathrm{t}} Z$. Es necesario extraer un número máximo de componentes en el ACM, es decir Min $\{\mathrm{m}, \mathrm{N}-1\}$, donde $\mathrm{m}$ representa la cantidad de categorías de aquellas variables sin datos faltantes menos el número de dichas variables y $\mathrm{N}$ representa el tamaño de la muestra. La distancia $\mathrm{X}^{2}$ entre dos modalidades es:

$$
d^{2}(j, k)=\sqrt{\sum_{i=1}^{n} n\left(\frac{X_{i j}}{n_{j}}-\frac{X_{i k}}{n_{k}}\right)^{2}}
$$

En la tabla de contingencia o Burt la contribución j-ésima modalidad a la inercia global con respecto al centro de gravedad es:

$$
\text { Contribución } I_{G C}(j)=p_{j} d_{(j, G c) \quad \forall j \varepsilon J}^{2}
$$

Obteniendo la inercia global con respecto a Gc de la nube de puntos $N(J)$ :

$$
I_{G c}(N(J))=\frac{K}{p}-1
$$

De modo que, depende del número de características observadas (p) y número de modalidades que presentan esas características (K) (Tsagris, 2016; Díaz, 2012). 


\section{MATERIAL Y MÉTODOS}

Población: Constituida por 54 estudiantes de estudios generales de la escuela de Estadística de la Universidad Nacional de Trujillo que registraron matrícula en el año 2019. Muestra: conformada por 34 estudiantes, para la cual se utilizó un muestreo aleatorio irrestricto:

$$
n=\left(\frac{z_{\alpha}+z_{\beta} \sqrt{1-r^{2}}}{r}\right)^{2}+2
$$

Donde, n: tamaño de muestra, $z_{\alpha}$ : nivel de confianza al $99 \%=2,33, z_{\beta}$ : función de potencia del $90 \%=1,28, \mathrm{r}$ : estimación de correlación = 0,6 (de muestra piloto).

Para la recolección de la información, se empleó como instrumento el cuestionario, el cual constó de 9 ítems referidos a información demográfica, social e institucional de los alumnos, al ser un instrumento con respuestas sin subjetividad, no requirió evaluación de confiabilidad. El rendimiento académico se obtuvo con el acta de notas proporcionado por la Dirección de Escuela. El diseño de investigación fue no experimental, transversal y descriptivo, $O \rightarrow G$. Las técnicas estadísticas empleadas fueron la selección de Clúster para la clasificación de variables y el Análisis Factorial de Correspondencias Múltiples para determinar el perfil de los estudiantes según su promedio ponderado empleando el software R-Studio.

\section{RESULTADOS}

\section{Tabla 1}

Frecuencia de factores asociados al rendimiento académico de los estudiantes de la escuela de Estadística, 2019

\begin{tabular}{|c|c|c|}
\hline & Frecuencia & Porcentaje \\
\hline \multicolumn{3}{|l|}{ Promedio Ponderado } \\
\hline PromP: 0 a 10 & 5 & $14,8 \%$ \\
\hline PromP: 11 a 14 & 23 & $67,6 \%$ \\
\hline PromP: 15 a 20 & 6 & $17,6 \%$ \\
\hline \multicolumn{3}{|l|}{ Porcentaje de Asistencia } \\
\hline Asistencia: $<70 \%$ & 9 & $26,5 \%$ \\
\hline Asistencia: > $70 \%$ & 25 & $73,5 \%$ \\
\hline \multicolumn{3}{|l|}{ Edad } \\
\hline Edad: $<20$ & 18 & $52,9 \%$ \\
\hline Edad: $>=20$ & 16 & $47,1 \%$ \\
\hline \multicolumn{3}{|l|}{ Sexo } \\
\hline Sexo: Fem & 12 & $35,3 \%$ \\
\hline Sexo: Masc & 22 & $64,7 \%$ \\
\hline \multicolumn{3}{|l|}{ Lugar de Residencia } \\
\hline Residencia: no_TRU_m & 5 & $14,7 \%$ \\
\hline Residencia: TRU_M & 29 & $85,3 \%$ \\
\hline \multicolumn{3}{|c|}{ Nivel Educativo más alto entre los padres } \\
\hline Niv_educ: PSec- & 15 & $44,1 \%$ \\
\hline Niv_educ: PSupT & 9 & $26,5 \%$ \\
\hline Niv_educ: PSupU & 10 & $29,4 \%$ \\
\hline
\end{tabular}




\begin{tabular}{lcc}
\hline Entor_fam: FExt & 3 & $8,8 \%$ \\
Entor_fam: FNuc & 27 & $79,4 \%$ \\
Entor_fam: Vivsolo & 4 & $11,8 \%$ \\
\hline Situación Laboral & & \\
\hline Laboral: NoTrab & 26 & $76,5 \%$ \\
Laboral: Trab & 8 & $23,5 \%$ \\
\hline Medio de Traslado & 6 & $17,6 \%$ \\
\hline Traslado: Pie & 6 & $3,0 \%$ \\
Traslado: TPriv & 1 & $79,4 \%$ \\
Traslado: TPub & 27 & \\
\hline Conformación de Grupos & & $41,2 \%$ \\
\hline Grupos: GHet & 14 & $58,8 \%$ \\
\hline Grupos: GHomo & 20 &
\end{tabular}

Fuente: Elaboración propia del autor

En primer lugar, la Tabla 1 muestra la variable promedio ponderado, donde el $14,8 \%$ se encuentra en el rango de 0 a 10; el 67,6 \%, de 11 a 14; y el otro 17,6\%, de 15 a 20 puntos, en una escala de notas de 0 a 20. En segundo lugar, la variable porcentaje de asistencia, donde el $26,5 \%$ presenta un porcentaje menor al $70 \%$ y el $73,5 \%$, mayor a ese valor. En tercer lugar, la variable edad, donde el $52,9 \%$ es menor a 20 años y el $47,1 \%$ es mayor o igual a 20 años. En cuarto lugar, la variable sexo, donde el 35,3\% son mujeres y el 64,7 $\%$, hombres. En quinto lugar, la variable lugar de residencia donde el 14,7 \% viven fuera de Trujillo Metropolitano y el 85,3 \%, dentro de Trujillo Metropolitano. En sexto lugar, la variable nivel educativo más alto entre los padres, donde el 44,1\% son de secundaria; el 26,5 \%, superior técnico; y el 29,4 \%, superior universitario. En séptimo lugar, la variable entorno familiar, donde el $8,8 \%$ han sido criados en una familia extendida; el $79,4 \%$, en una familia nuclear; y el 11,8 \% viven solos. En octavo lugar, la variable situación laboral, donde el 76,5 \% no trabajan y el 23,5 \% si lo hacen. En noveno lugar, la variable medio de traslado, donde el $17,6 \%$ se trasladan a pie; el $3 \%$, en transporte privado; y el 79,4 \%, en transporte público. En décimo lugar, la conformación de grupos, donde el 41,2 \% prefieren grupos heterogéneos y el 58,8 \%, grupos homogéneos. Por último, se tiene la variable presencia de cursos rezagados, donde el 76,5\% no presentan cursos rezagados y el 23,5 \% si presentan.

\section{Figura 1}

Dendograma de factores asociados al rendimiento académico

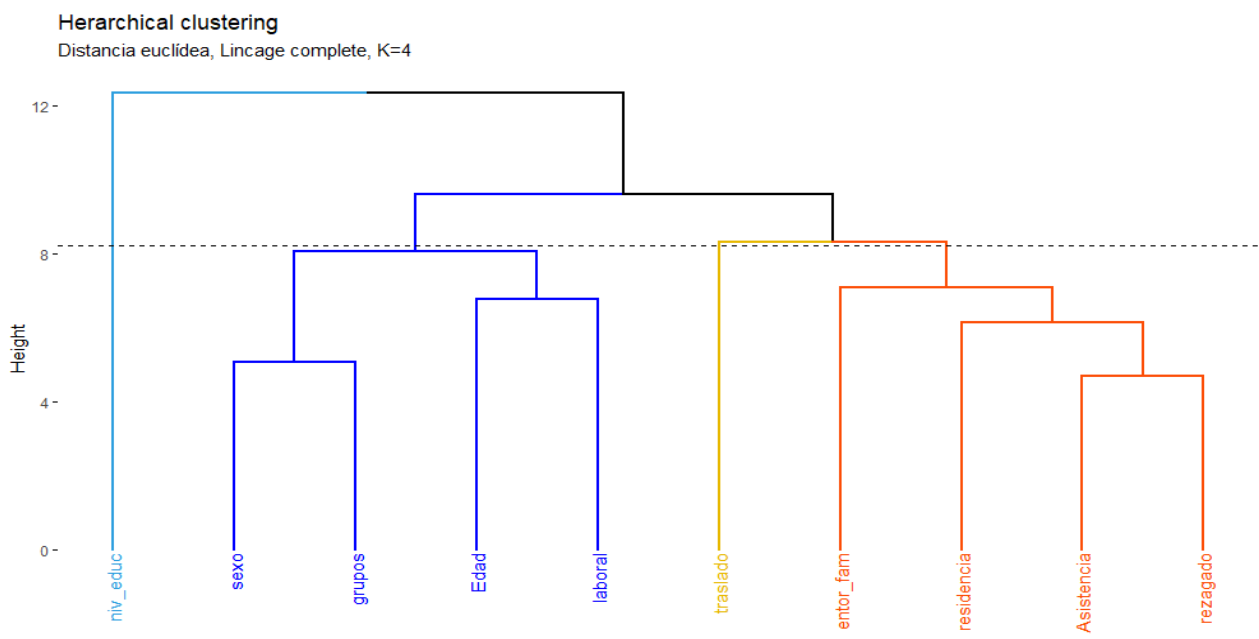


En la Figura 1 se determinó con un punto de corte igual a ocho 4 clúster denominados: Nivel educativo más alto entre los padres del estudiante, Factores sociodemográficos, Medio de traslado a la Universidad Nacional de Trujillo y Factores de distancia y responsabilidad académica. En las Figuras 2, 3 y 4, se pueden observar los acercamientos del plano factorial de asociación de las variables del estudio, donde se determinó el perfil de los estudiantes según su promedio ponderado en la escala del 0 al 20; los estudiantes que se encuentran en el rango de 0 a 10 puntos, se caracterizan por no vivir en Trujillo Metropolitano, presentan uno o más cursos rezagados, su medio de traslado a la universidad es a pie y son menores de 20 años; los estudiantes que han obtenido un promedio ponderado en el rango de 15 a 20 puntos, se caracterizan por el nivel de estudios más alto entre sus padres que es superior universitario, tienen una familia extensa, su asistencia es mayor al $70 \%$ y no trabajan; y por último los estudiantes que han obtenido un promedio ponderado en el rango de 11 a 14 puntos, se caracterizan por su conformación de grupos que es heterogénea, sexo femenino, no presentan cursos rezagados, su medio de traslado es el transporte público, viven en Trujillo Metropolitano y tienen una edad mayor o igual a 20 años.

\section{Figura 2}

Primer acercamiento al plano factorial

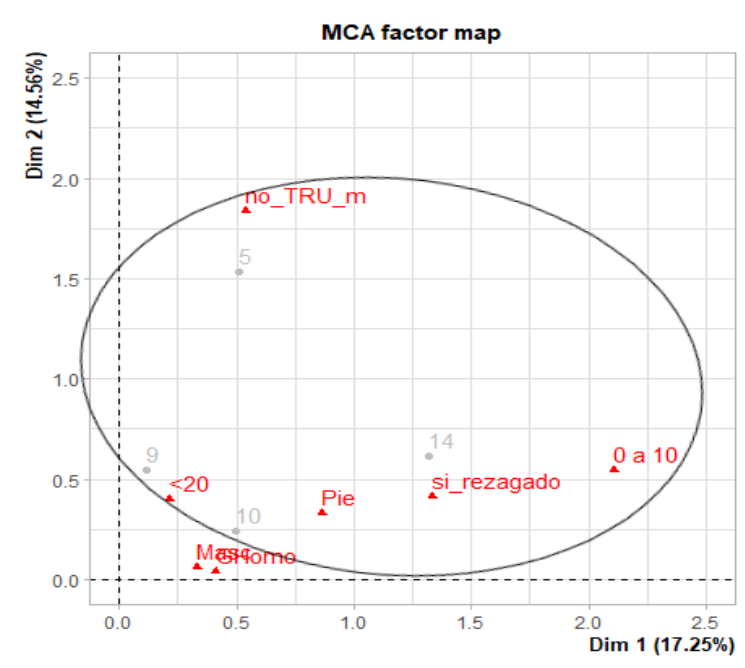




\section{Figura 3}

Segundo acercamiento al plano factorial

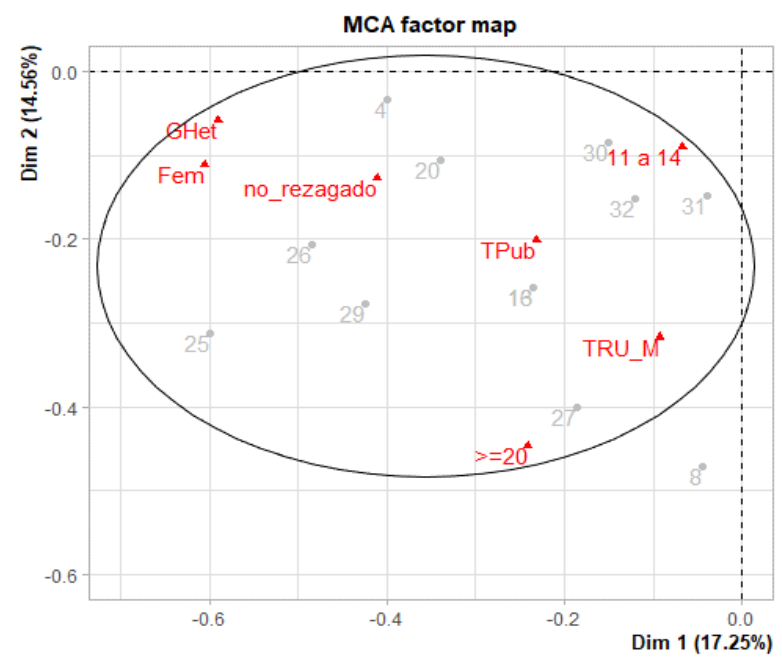

Figura 4

Tercer acercamiento al plano factorial

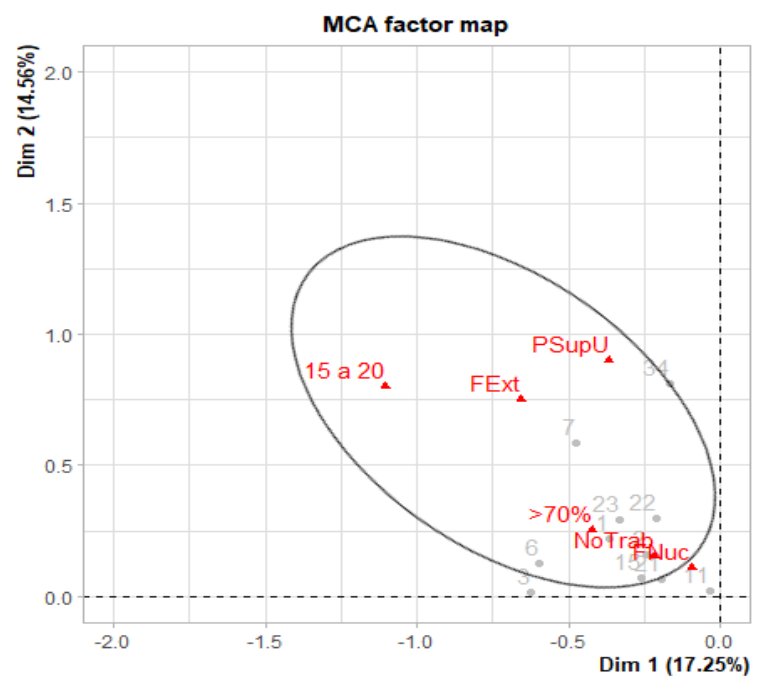

\section{DISCUSIÓN}

El presente trabajo se enfocó en la obtención de factores asociados al rendimiento académico de los estudiantes de estudios generales de la carrera de Estadística a partir de un conjunto de variables para posteriormente agruparlas en factores y determinar el perfil del estudiante según los niveles del rendimiento académico obtenidos a partir de su promedio ponderado; lo cual difiere con Garbanzo (2007), quien parte desde factores ya clasificados en tres categorías: factores personales, sociales e institucionales.

En la tesis de Solano (2015), se indicó que existe relación significativa entre la motivación de asistir a clases y el rendimiento; determinándose un coeficiente de 0,560 ; coincidiendo con nuestro estudio al evaluar la relación entre los promedios ponderados y el porcentaje de asistencia a la universidad de los estudiantes de estadística con un coeficiente de 0,6. Asimismo, en los estudiantes de Ingeniería Estadística sus promedios ponderados según 
el sexo no varían, ambos puntúan de manera similar, los hombres presentan un promedio general de 12,35 y las mujeres 12,21 encontrándose en el rango de 11 a 14, lo cual coincide con el estudio de Tineo (2015), donde se llegó a la misma conclusión, que tanto mujeres como varones tienen una puntuación promedio similar.

Por último, Nuñez y Hernández (2017) realizaron la prueba $\chi^{2}$ de Pearson para medir la relación del rendimiento académico con el nivel educativo de los padres, siendo significativa para la madre, más no para el padre. Con la información disponible para los estudiantes de estudios generales de Ingeniería Estadística se replicó la prueba utilizada, encontrando que no existe relación significativa entre el nivel educativo más alto entre los padres y/o apoderado del estudiante, con su rendimiento académico con un p-valor > 0,05 , coincidiendo con el estudio anterior respecto al nivel educativo del padre.

\section{CONCLUSIONES}

Los factores encontrados que se asocian al rendimiento académico de los estudiantes de estadística pueden clasificarse en cuatro clústeres: Nivel educativo más alto entre los padres del estudiante, Factores sociodemográficos; Medio de traslado a la universidad y los Factores de distancia y responsabilidad académica.

El perfil de los estudiantes con un promedio ponderado en el rango de 0 a 10 , se caracteriza por: no vivir en Trujillo Metropolitano, presentan uno o más cursos rezagados, su medio de traslado a la universidad es a pie y son menores de 20 años. El perfil de los estudiantes con un promedio ponderado en el rango de 15 a 20, se caracteriza por: el nivel de estudios más alto entre sus padres que es superior universitario, tienen una familia extensa, su asistencia es mayor al $70 \%$ y no trabajan.

El perfil de los estudiantes con un promedio ponderado de 11 a 14 se caracteriza por: su conformación de grupos que es heterogénea, sexo femenino, no presentan cursos rezagados, su medio de traslado es el transporte público, viven en Trujillo Metropolitano y tienen una edad mayor o igual a 20 años.

\section{RECOMENDACIONES}

Se recomienda a toda la comunidad universitaria tomar como referencia este estudio para que se lleven a cabo más investigaciones con otras facultades y escuelas, ampliando las variables que se consideró en esta tesis, con la intención de determinar otros posibles factores que afecten al rendimiento académico, para poder abarcar el problema en su totalidad. Proponiendo así soluciones de mejora que logre el bienestar de los estudiantes, teniendo en cuenta el nuevo currículo de enseñanza superior basada en competencias, donde el estudiante es el principal protagonista en la educación y su bienestar emocional es de vital importancia.

Se propone a las autoridades y docentes de la escuela de Estadística, tomar como referente esta investigación para mejorar el rendimiento académico de sus estudiantes, trazando novedosas estrategias educativas para lograr la motivación del alumnado. En tal sentido, se propone la realización de capacitaciones para entender la importancia de conocer los factores asociados al rendimiento académico, que de una u otra manera afectan el rendimiento académico del universitario. 
Se sugiere a la dirección de escuela de Estadística estar informados de la situación laboral, familiar, etc. de los estudiantes, para poder orientarlos en la cantidad de cursos a matricularse, con el fin que puedan cumplir exitosamente con sus responsabilidades académicas.

\section{REFERENCIAS}

Abarca, R. (2019). La UNSA retira a 118 alumnos por bajo rendimiento académico. Correo. Recuperado de https://diariocorreo.pe/edicion/arequipa/la-unsa-retira118-alumnos-por-bajo-rendimiento-academico-909748/

Chong, E. (2017). Factores que inciden en el rendimiento académico de los estudiantes de la Universidad Politécnica del Valle de Toluca. Revista Latinoamericana de Estudios Educativos, XLVII(1). Disponible en: https://www.redalyc.org/articulo.oa?id=270/27050422005

Díaz, L. (2012). Estadística multivariada: inferencia y métodos. Bogotá, Colombia: Editorial Universidad Nacional de Colombia

Echevarría, J. (2013). Causas del bajo rendimiento académico universitario en los alumnos del tercer año de la Facultad de Derecho y Ciencias Políticas de la Universidad Nacional de Trujillo en el año 2013 (tesis de licenciatura).

Garbanzo, G. (2010). Factores Asociados al Rendimiento académico en estudiantes universitarios. Costa Rica.

Hernández, N. y Nuñez, C. (2017). Influencia del rendimiento previo en el rendimiento académico y su relación con el nivel socio económico en estudiantes de ciencias de la salud de una universidad de Cajamarca (tesis de postgrado).

Hernández, R. (2010). Psicología de la Educación. México: Ediciones Trillas.

MINEDU (2016). El Perú se caracteriza por las mínimas posibilidades que tiene su población para permitirse una educación superior. Correo. Recuperado de http://www.minedu.gob.pe/politicas/docencia/bienestar-docente.php

Muradás, M (2016). Diagnóstico de algunos factores de riesgo asociados a la deserción estudiantil de los alumnos de la Universidad Politécnica del Centro. Disponible en: http://www.revistas.ujat.mx/index.php/perspectivas/article/view/1307

Penta Analytics (2017). El $27 \%$ de ingresantes a universidades privadas abandonan su carrera en primer año de estudios. Gestión. Recuperado de: https://gestion.pe/tendencias/management-empleo/27-ingresantes-universidadesprivadas-abandonan-carrera-primer-ano-estudios-139168-noticia/

Sánchez, L. (2017). Factores que inciden en el rendimiento académico de los estudiantes de Ingeniería Industrial. Lima

Tsagris, M (2016). Multivariate data análisis in $R, A$ collection of $R$ functions for multivariate data analysis. Herakleion, Grecia: Department of Computer Science, University of Crete.

Vidal, R (2018). Técnicas multivariantes de interdependencia, casos reales y prácticos de investigación. Madrid, España: Ra-Ma Editorial. 